\title{
BODY FORCE WEIGHT FUNCTIONS OF CRACKED BODIES
}

\author{
CHIEN-CHING MA and MIN-HWA LIAO \\ Department of Mechanical Engineering, National Taiwan University, Taipei, Taiwan 10764. \\ Republic of China
}

\begin{abstract}
Most of the investigations which calculate the stress intensity factor by using the weight function concept all focus on the problem with traction boundary conditions. In this study, the expression of body force weight functions in a two-dimensional cracked body is formulated. Stress intensity factors can be calculated easily for any arbitrary distribution of body force from the predetermined weight functions. Body force weight functions for rectangular cracked plates with finite dimensions are calculated by the numerical boundary element method. Some practical examples of various crack configurations and boundary conditions are given and a very satisfactory accuracy is obtained. It should be noted that the ordinary traction type of weight function is a special case of the body force weight function discussed in this study.
\end{abstract}

\section{INTRODUCTION}

THE WEIGHT function method which was first proposed by Bueckner [1] is a powerful and efficient method for determining the stress intensity factor for the mechanical loading system. In his formulation, the weight function is the displacement of the fundamental state induced by a self-equilibrating loading for which the singularity is one order higher than the normal state. Rice [2] proposed a convenient formulation for the determination of the weight function, which then became the most prevailing form. He showed that if the displacement field $u^{(1)}(s, a)$ and mode I stress intensity factor $K_{I}^{(1)}$ are known as functions of crack length for any loading system "l", then the mode I stress intensity factor $K_{\mathrm{I}}^{(2)}$ for any other loading $t^{*(2)}$ of system " 2 " acting on the same body has the following form

$$
K_{\mathrm{I}}^{(2)}=\frac{H}{2 K_{1}^{(1)}} \int t^{*(2)} \frac{\partial u^{(1)}}{\partial a} \mathrm{~d} s
$$

where $H=E$ for plane stress, $H=E /\left(1-v^{2}\right)$ for plane strain, $v$ being Poisson's ratio and $a$ the crack length. The weight function $h_{1}^{S t}(s, a)$ on the traction boundary is defined as

$$
h_{\mathrm{1}}^{S t}(s, a)=\frac{H}{2 K_{\mathrm{l}}^{(1)}} \frac{\partial u^{(1)}}{\partial a} .
$$

The weight function is a universal function for a given crack geometry and composition, and is independent of applied loading. If the weight function is obtained from a single simple loading case, it can then be used to calculate stress intensity factors for any other complicated loading system of the same cracked geometry.

Freund and Rice [3] used the Laplace transform and extended the weight function concept for dynamic stress fields in an elastic solid containing a crack. Lately, Bowie and Freese [4] have made a reformulation for the weight function to include the mixed traction and displacement boundary conditions. In the study of Bortman and Banks-Sills [5], Rice's definition of the weight function for mode I has been extended to mixed fracture mode. Sham and Zhou [6] investigated the weight functions in anisotropic bodies under plane deformation. The extension of the weight function theory to the three-dimensional regime was derived by Rice [2] and Bueckner [7]. The developments of the three-dimensional weight functions can be found in the articles of Rice [8,9], Bueckner [10], Sham [11], Sham and Zhou [12], Gao [13, 14], and Gao and Rice [15]. Recently, Ma et al. [16] used the finite element method to determine weight functions in a two-dimensional mixed mode cracked body with finite dimensions. Explicit crack-face weight functions are presented for plates containing edge and center cracks by using the least squares fitting procedure. 


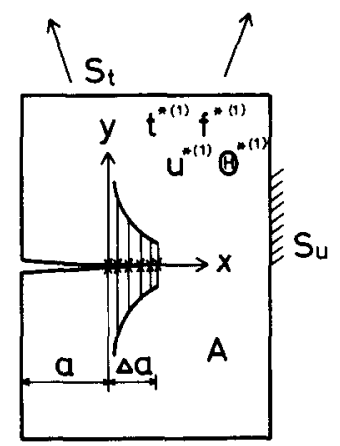

(1)

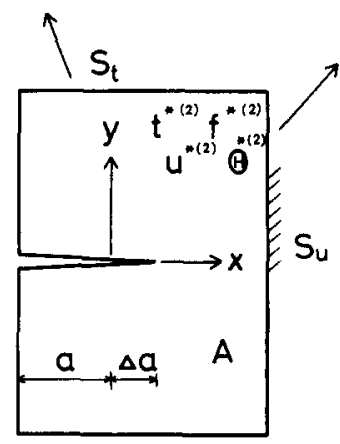

(2)

Fig. 1. Configuration of a cracked body subjected to loading system " 1 " and " 2 ".

Most of the investigations on the weight function mentioned above are focused on the boundary on which the surface traction is applied. Our purpose here is to extend the weight function concept for mechanical loading applied at the traction boundary to the body force applied inside an elastic solid containing a crack. The boundary element method is used to construct the weight functions for a rectangular cracked plate by coupling the virtual crack extension technique. These predetermined weight functions can then be used to calculate the stress intensity factors induced by distributed body forces.

\section{WEIGHT FUNCTION FORMULATIONS}

Following the methodologies of Bowie and Freese [4], and Bortman and Banks-Sills [5], Tsai and $\mathrm{Ma}$ [17] extended the formulation of the weight function to include thermal loading by using Betti's reciprocal theorem. Consider the equivalent configurations "1" and "2" (Fig. 1). There are different sorts of loading type, such as prescribed traction $t^{*}$ at boundary $S_{t}$, prescribed displacement $\mathbf{u}^{*}$ at boundary $S_{u}$, body force $\mathbf{f}^{*}$ and temperature $\Theta^{*}$ at domain $A$. The applied traction $\mathbf{t}^{*(1)}$ in the vicinity of the crack tip is set to be the equilibrating traction which makes the relative displacement vanish along the crack face $\Delta a$. The loading system " 1 " is a known referential system. In order to obtain the proper formulation, the prescribed loading of system " 1 " is independent of crack length $a$, but the loading system " 2 " of interest has no such restriction.

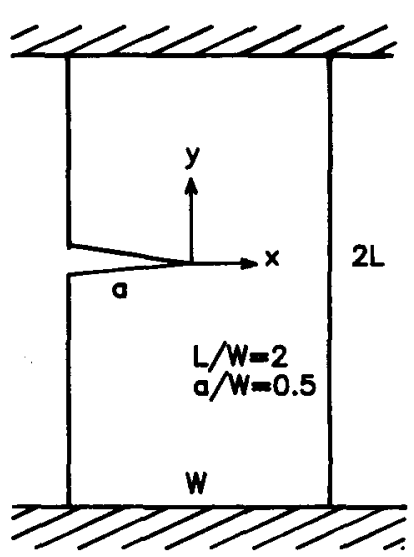

Fig. 2. Geometry and coordinate definitions for an edge crack with fixed boundary conditions. 


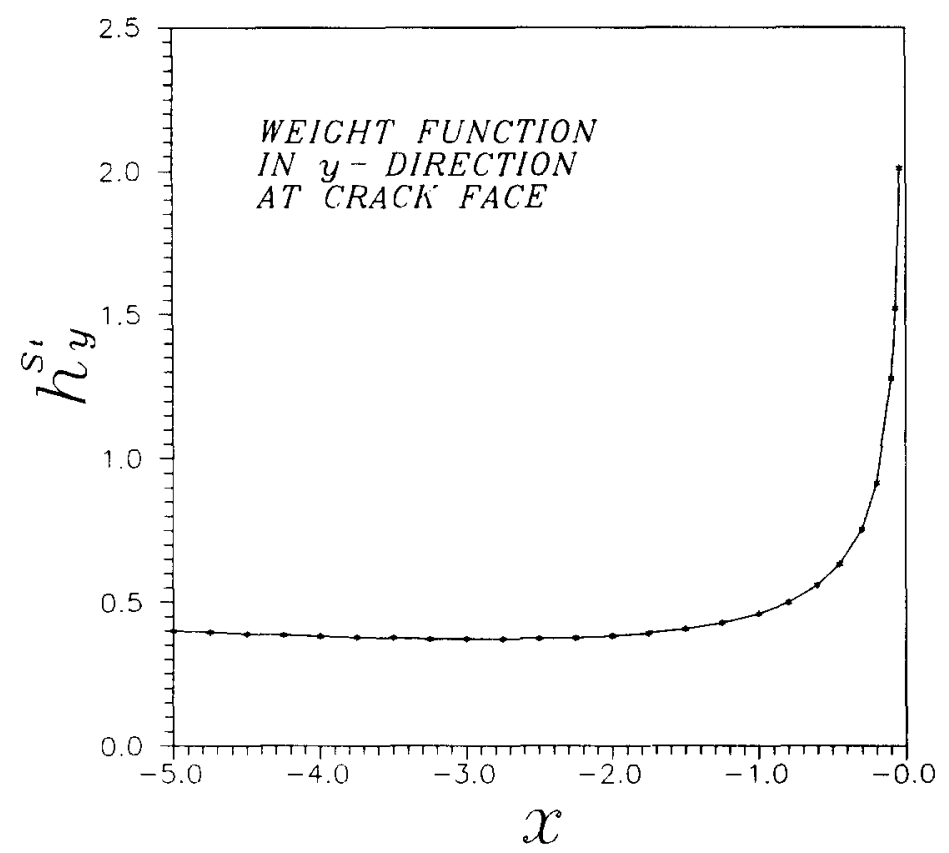

Fig. 3. Crack face weight functions for the edge crack as shown in Fig. 2.

First, let $\Delta a$ vanish in the two systems. Then, from Betti’s reciprocal theorem,

$$
\begin{aligned}
\int_{S_{t}} \mathbf{t}^{*(1)} \cdot \mathbf{u}^{(2)}(a) \mathrm{d} S+\int_{S_{u}} \mathbf{u}^{*(2)}(a) \cdot \mathbf{t}^{(1)}(a) \mathrm{d} S+\int_{A} \mathbf{f}^{*(1)} \cdot \mathbf{u}^{(2)}(a) \mathrm{d} A+\int_{A} \alpha \Theta^{*(1)} \sigma_{k k}^{(2)}(a) \mathrm{d} A \\
=\int_{S_{t}} \mathbf{t}^{*(2)}(a) \cdot \mathbf{u}^{(1)}(a) \mathrm{d} S+\int_{S_{u}} \mathbf{u}^{*(1)} \cdot \mathbf{t}^{(2)}(a) \mathrm{d} S+\int_{A} \mathbf{f}^{*(2)}(a) \cdot \mathbf{u}^{(1)}(a) \mathrm{d} A+\int_{A} \alpha \Theta^{*(2)}(a) \sigma_{k k}^{(1)}(a) \mathrm{d} A,
\end{aligned}
$$

where $\alpha$ is the coefficient of thermal expansion and $\sigma_{k k}$ is the sum of normal stresses $\sigma_{x x}+\sigma_{y y}$ for a two-dimensional problem. The surface traction $t$, displacement $u$ and body force $f$ expressed in eq. (3) are all vector quantities. Let $S_{\Delta a}$ denote the boundary of the extended crack surface $\Delta a$ and apply the reciprocal theorem to the geometry with crack length $a+\Delta a$, then

$$
\begin{aligned}
& \int_{S_{\Delta a}} \mathbf{t}^{*(1)} \cdot \mathbf{u}^{(2)}(a+\Delta a) \mathrm{d} S+\int_{S_{t}} \mathbf{t}^{*(1)} \cdot \mathbf{u}^{(2)}(a+\Delta a) \mathrm{d} S+\int_{S_{u}} \mathbf{u}^{*(2)}(a+\Delta a) \cdot \mathbf{t}^{(1)} \mathrm{d} S+\int_{A} \mathbf{f}^{*(1)} \cdot \mathbf{u}^{(2)}(a+\Delta a) \mathrm{d} A \\
& \quad+\int_{A} \alpha \Theta^{*(1)} \sigma_{k k}^{(2)}(a+\Delta a) d A=\int_{S_{t}} \mathbf{t}^{*(2)}(a+\Delta a) \cdot \mathbf{u}^{(1)}(a) \mathrm{d} S+\int_{S_{u}} \mathbf{u}^{*(1)} \cdot \mathbf{t}^{(2)}(a+\Delta a) \mathrm{d} S \\
& \quad+\int_{A} \mathbf{f}^{*(2)}(a+\Delta a) \cdot \mathbf{u}^{(1)}(a) \mathrm{d} A+\int_{A} \alpha \Theta^{*(2)}(a+\Delta a) \sigma_{k k}^{(1)}(a) \mathrm{d} A
\end{aligned}
$$

Differentiating eq. (3) with respect to $a$ and rewriting eq. (4) by the Taylor expansion yields the desired relationship

$$
\frac{1}{\Delta a} \int_{S_{\Delta a}} \mathbf{t}^{*(1)} \cdot \mathbf{u}^{(2)} \mathrm{d} S=-\int_{S_{t}} \mathbf{t}^{*(2) \cdot} \cdot \frac{\partial \mathbf{u}^{(1)}}{\partial a} \mathrm{~d} S+\int_{S_{u}} \mathbf{u}^{*(2) \cdot} \frac{\partial \mathbf{t}^{(1)}}{\partial a} \mathrm{~d} S-\int_{A} \mathbf{f}^{*(2) \cdot} \frac{\partial \mathbf{u}^{(1)}}{\partial a} \mathrm{~d} A-\int_{A} \alpha \Theta^{*(2)} \frac{\partial \sigma_{k k}^{(1)}}{\partial a} \mathrm{~d} A
$$


For the mixed mode condition, $\mathbf{t}^{*(1)}$ and $\mathbf{u}^{*(2)}$ in the vicinity of the crack tip can be represented as follows

$$
\begin{aligned}
& \left\{\begin{array}{l}
\mathbf{t}^{*(1)}\left(\chi, 0^{+}\right)=\left(\frac{-K_{l !}^{(1)}}{\sqrt{2 \pi x}}, \frac{-K_{1}^{(1)}}{\sqrt{2 \pi x}}\right) \\
\mathbf{t}^{*(1)}\left(\chi, 0^{-}\right)=\left(\frac{\mathbf{K}_{11}^{(1)}}{\sqrt{2 \pi x}}, \frac{K_{1}^{1)}}{\sqrt{2 \pi x}}\right)
\end{array}\right. \\
& \begin{cases}\mathbf{u}^{(2)}\left(\chi, 0^{+}\right)=\left(\frac{4 \mathrm{~K}_{1}^{(2)}}{\sqrt{2 \pi} H} \sqrt{\Delta a-x},\right. & \left.\frac{4 K_{1}^{(2)}}{\sqrt{2 \pi} H} \sqrt{\Delta a-x}\right) \\
\mathbf{u}^{(2)}\left(\chi, 0^{-}\right)=\left(\frac{-4 \mathrm{~K}_{11}^{(2)}}{\sqrt{2 \pi} H} \sqrt{\Delta a-x},\right. & \left.\frac{-4 K_{1}^{(2)}}{\sqrt{2 \pi} H} \sqrt{\Delta a-x}\right) .\end{cases}
\end{aligned}
$$

Substitution of these near tip fields into eq. (5) yields the following relationship

$$
K_{1}^{(1)} K_{!}^{(2)}+K_{11}^{(1)} K_{11}^{(2)}=\frac{H}{2}\left(\int_{S_{t}} \mathbf{t}^{*(2)} \cdot \frac{\partial \mathbf{u}^{(1)}}{\partial a} \mathrm{~d} S-\int_{S_{u}} \mathbf{u}^{*(2) \cdot} \cdot \frac{\partial \mathbf{t}^{(1)}}{\partial a} \mathrm{~d} S+\int_{A} \mathbf{f}^{*(2)} \cdot \frac{\partial \mathbf{u}^{(1)}}{\partial a} \mathrm{~d} A+\int_{A} \alpha \Theta^{*(2)} \frac{\partial \sigma_{k k}^{(1)}}{\partial a} \mathrm{~d} A\right)
$$

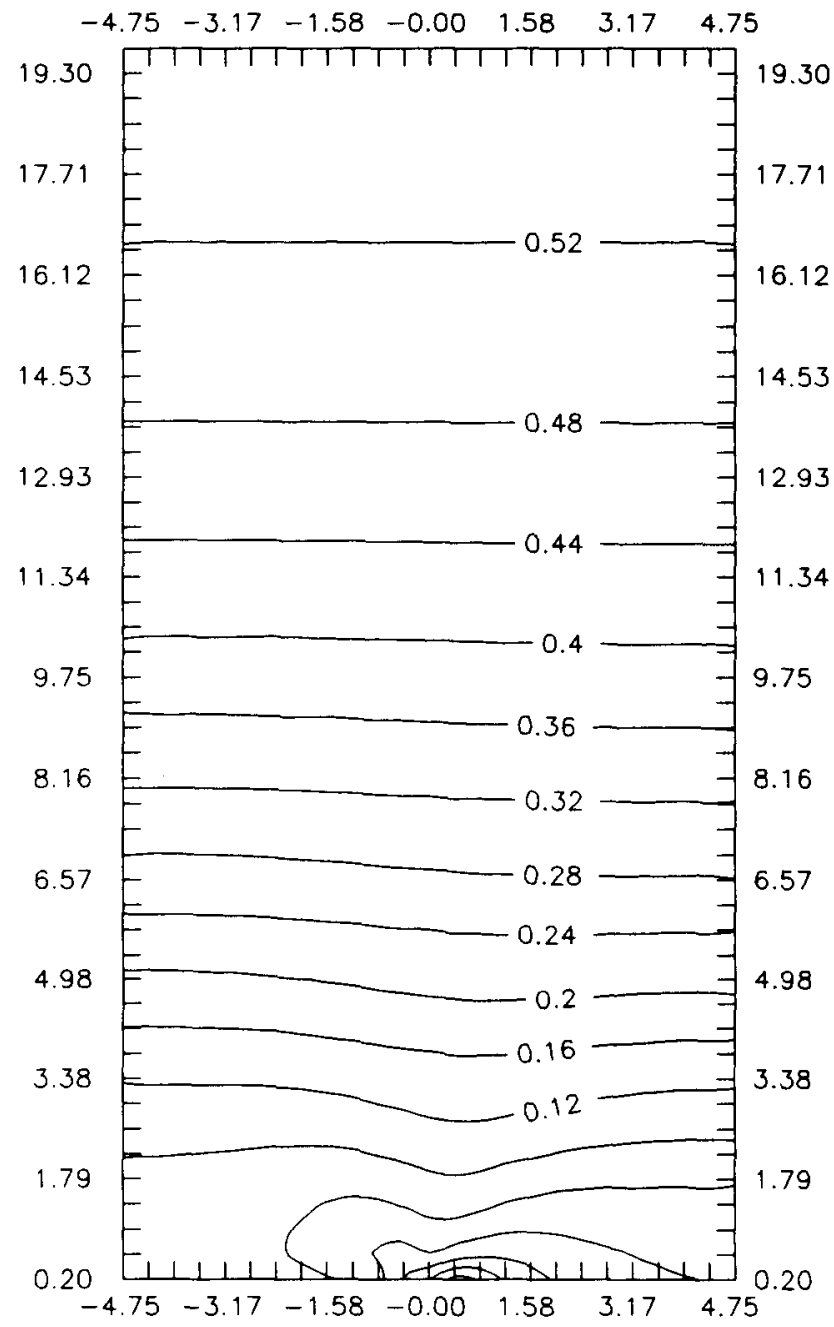

Fig. 4. The body force weight function for applying body force in the $x$-direction. 


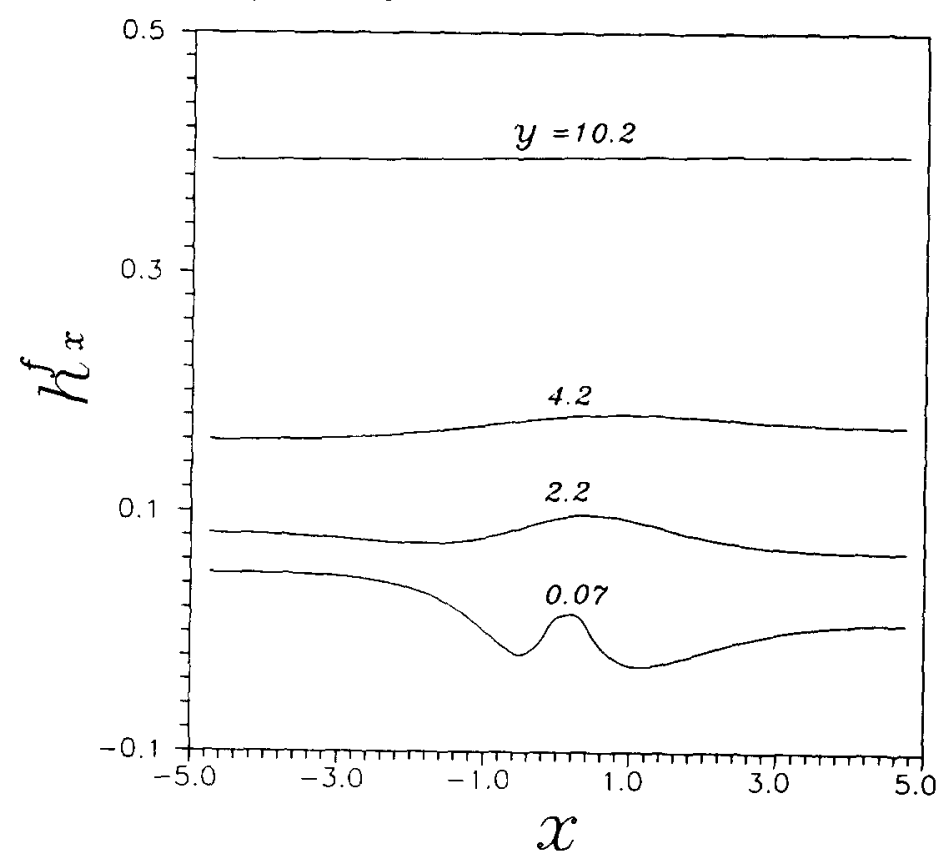

Fig. 5. The variation of the body force weight function as a function of $x$ for different values of $y$.

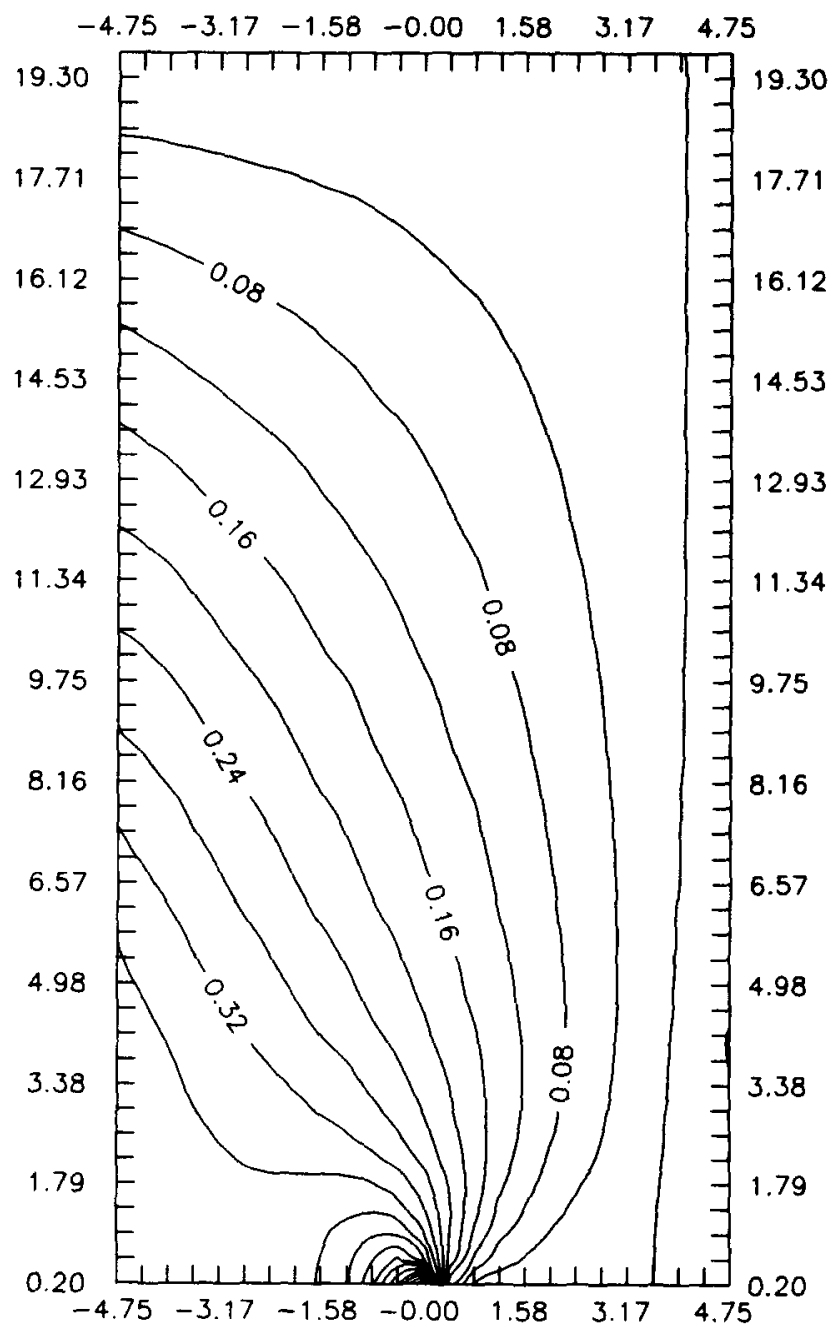

Fig. 6. The body force weight function for applying body force in the $y$-direction. 


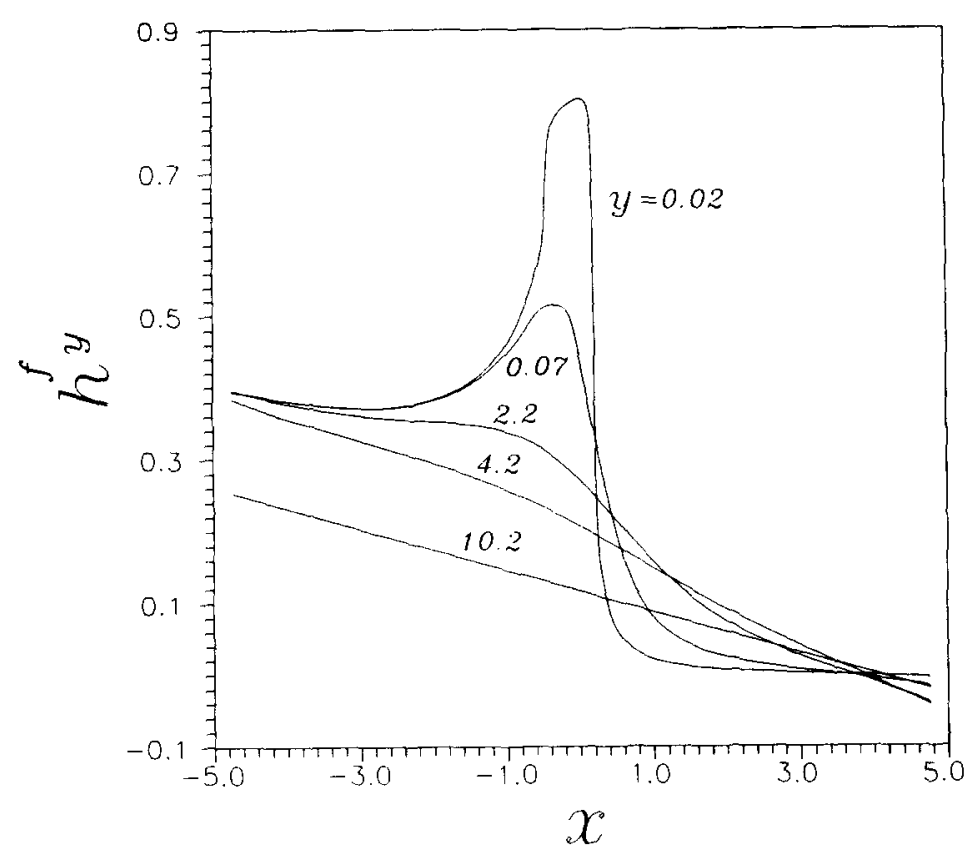

Fig. 7. The variation of the body force weight function as a function of $x$ for different values of $y$.

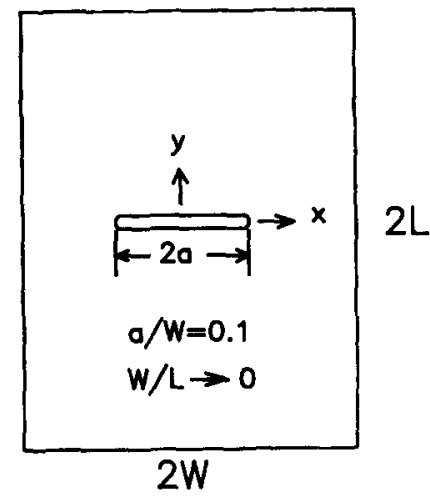

Fig. 8. Geometry and coordinate definitions for a center crack with traction boundary conditions.

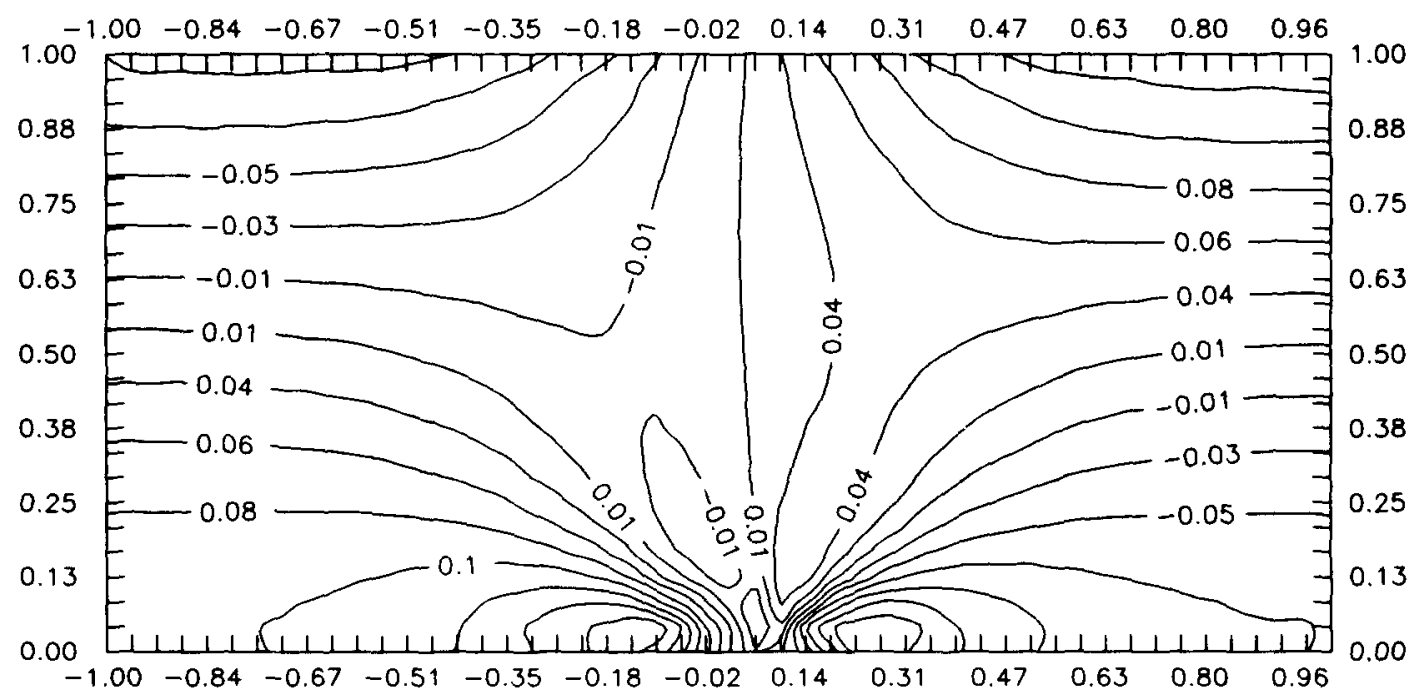

Fig. 9. The body force weight function for applying body force in the $x$-direction of the center cracked plate. 


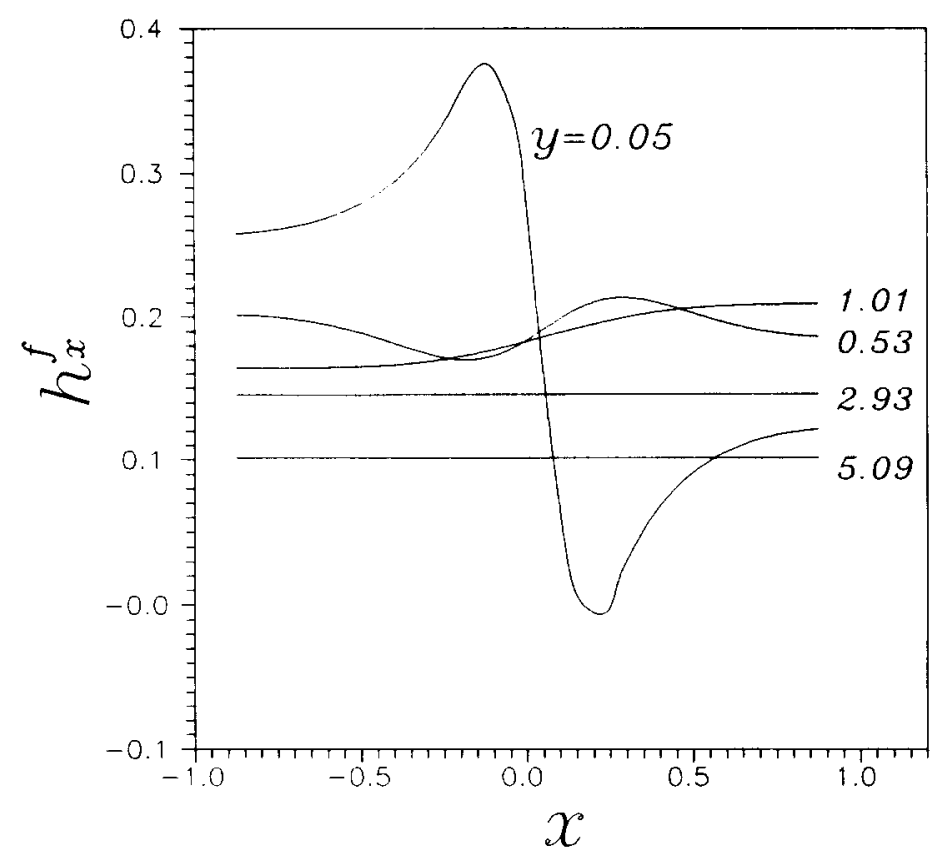

Fig. 10. The variation of the body force weight function as a function of $x$ for different values of $y$.

If another loading system " 3 " is introduced, then from the similar procedure, we have

$K_{1}^{(3)} K_{1}^{(2)}+K_{\mathrm{II}}^{(3)} K_{\mathrm{II}}^{(2)}=\frac{H}{2}\left(\int_{S_{t}} \mathbf{t}^{*(2)} \cdot \frac{\partial \mathbf{u}^{(3)}}{\partial a} \mathrm{~d} S-\int_{S_{u}} \mathbf{u}^{*(2)} \cdot \frac{\partial \mathbf{t}^{(3)}}{\partial a} \mathrm{~d} S+\int_{A} \mathbf{f}^{*(2)} \cdot \frac{\partial \mathbf{u}^{(3)}}{\partial a} \mathrm{~d} A+\int_{A} \alpha^{*(2)} \frac{\partial \sigma_{k k}^{(3)}}{\partial a} \mathrm{~d} A\right)$.

From eqs (8) and (9), the general expressions for the mixed-mode stress intensity factors $K_{1}^{(2)}$ and $K_{11}^{2)}$ subjected to the above-mentioned loadings can be expressed as follows

$$
\begin{aligned}
K_{\mathrm{I}}^{(2)}=\frac{H}{2 K} & {\left[\int_{S_{t}} \mathbf{t}^{*(2)} \cdot\left(K_{\mathrm{II}}^{(3)} \frac{\partial \mathbf{u}^{(1)}}{\partial a}-K_{\mathrm{H}}^{(1)} \frac{\partial \mathbf{u}^{(3)}}{\partial a}\right) \mathrm{d} S-\int_{S_{u}} \mathbf{u}^{*(2)} \cdot\left(K_{\mathrm{II}}^{(3)} \frac{\partial \mathbf{t}^{(1)}}{\partial a}-K_{\mathrm{HI}}^{(1)} \frac{\partial \mathbf{t}^{(3)}}{\partial a}\right) \mathrm{d} S\right.} \\
& \left.+\int_{A} \mathbf{f}^{*(2)} \cdot\left(K_{\mathrm{II}}^{3)} \frac{\partial \mathbf{u}^{(1)}}{\partial a}-K_{\mathrm{II}}^{(1)} \frac{\partial \mathbf{u}^{(3)}}{\partial a}\right) \mathrm{d} A+\int_{A} \alpha \Theta^{*(2)}\left(K_{\mathrm{II}}^{(3)} \frac{\partial \sigma_{k k}^{(1)}}{\partial a}-K_{\mathrm{ll}}^{(1)} \frac{\partial \sigma_{k k}^{(3)}}{\partial a}\right) \mathrm{d} A\right],
\end{aligned}
$$

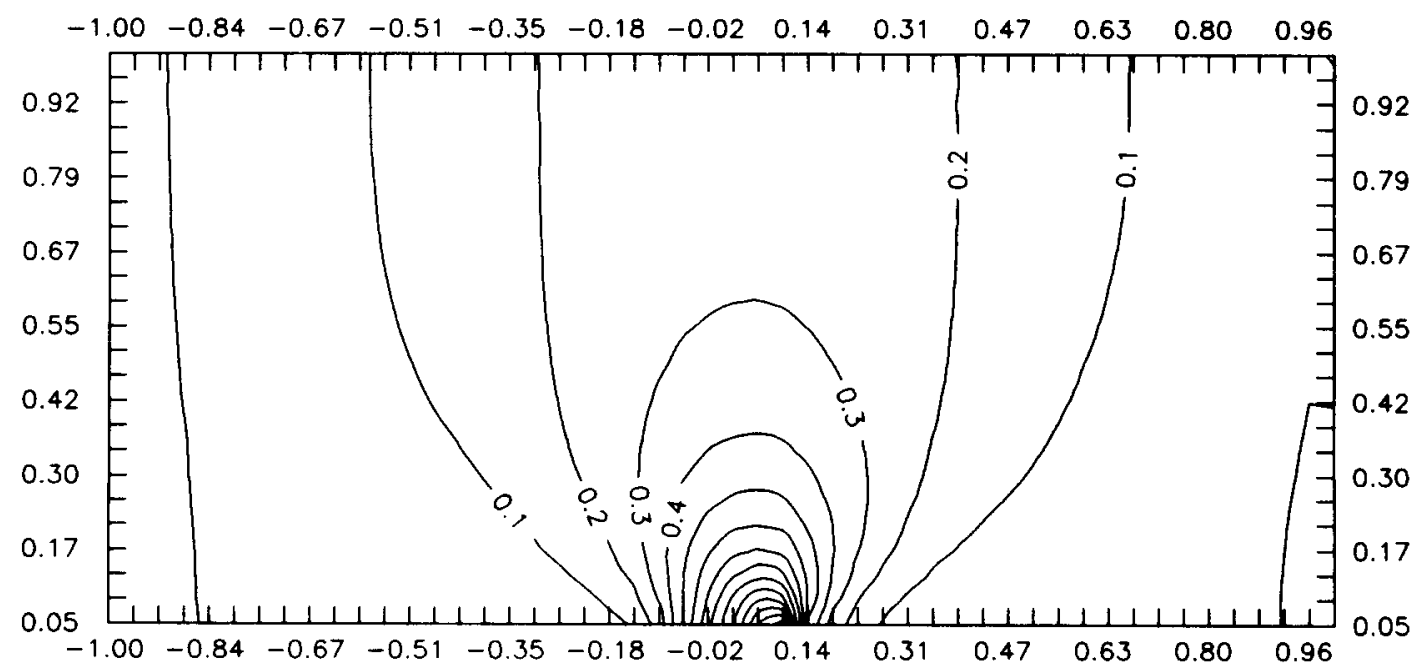

Fig. 11. The body force weight function for applying body force in the $y$-direction of the center cracked plate. 


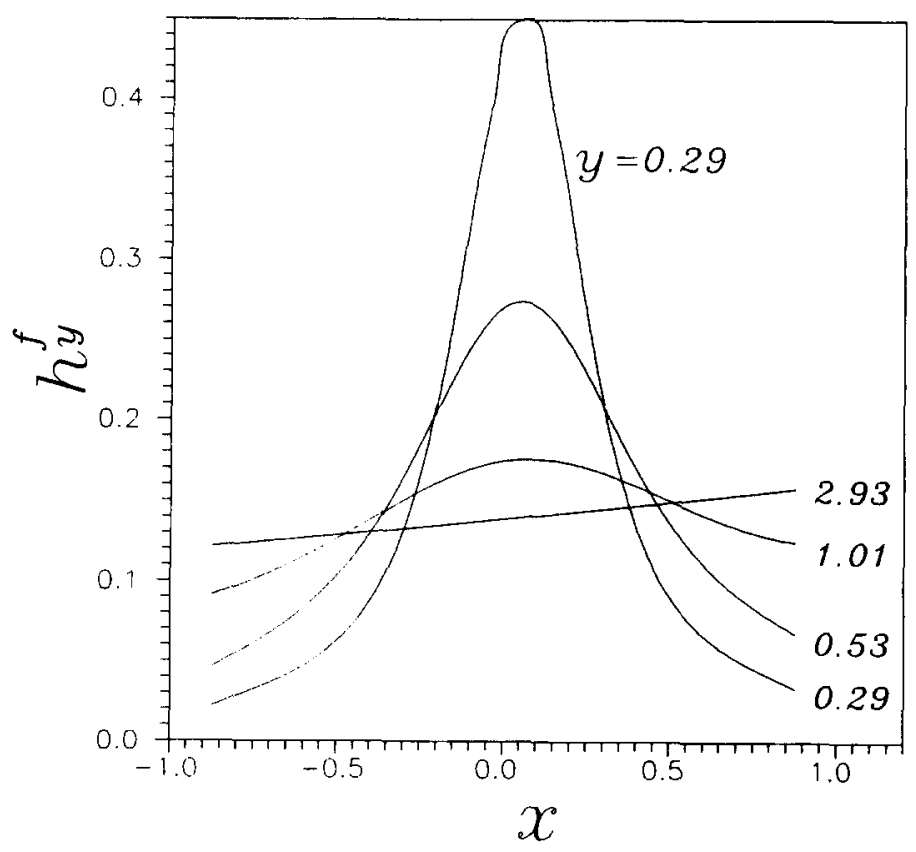

Fig. 12. The variation of the body force weight function as a function of $x$ for different values of $y$.

$$
\begin{aligned}
& K_{I I}^{(2)}=\frac{H}{2 K}\left[\int_{S_{t}} \mathbf{t}^{*(2) \cdot}\left(K_{\mathrm{l}}^{(1)} \frac{\partial \mathbf{u}^{(3)}}{\partial a}-K_{1}^{(3)} \frac{\partial \mathbf{u}^{(1)}}{\delta a} \partial a\right) \mathrm{d} S-\int_{S_{u}} \mathbf{u}^{*(2)} \cdot\left(K^{(1)} \frac{\partial \mathbf{t}^{(3)}}{\partial a}-K_{\mathrm{f}}^{(3)} \frac{\partial \mathbf{t}^{(1)}}{\partial a}\right) \mathrm{d} S\right. \\
& \left.+\int_{A} \mathbf{f}^{*(2)}\left(K_{\uparrow}^{(1)} \frac{\partial \mathbf{u}^{(3)}}{\partial a}-K_{\uparrow}^{3)} \frac{\partial \mathbf{u}^{(1)}}{\partial a}\right) \mathrm{d} A+\int_{A} \alpha \Theta^{*(2)}\left(K_{l}^{(1)} \frac{\partial \sigma_{k k}^{(3)}}{\partial a}-K_{\uparrow}^{(3)} \frac{\partial \sigma_{k k}^{(1)}}{\partial a}\right) \mathrm{d} A\right]
\end{aligned}
$$

where $K=K_{1}^{1)} K_{11}^{(3)}-K_{1}^{(3)} K_{11}^{(1)}$. The loading systems "1" and "3" are known as arbitrary referential systems, while the loading system " 2 " is the one that we are interested in. If only the body force is considered, we have

$$
\begin{aligned}
& K_{\mathrm{f}}^{(2)}=\frac{H}{2 K} \int_{A} \mathbf{f}^{*(2) \cdot}\left(K_{\mathrm{fI}}^{(3)} \frac{\partial \mathbf{u}^{(1)}}{\partial a}-K_{\mathrm{fl}}^{(1)} \frac{\partial \mathbf{u}^{(3)}}{\partial a}\right) \mathrm{d} A, \\
& K_{\mathrm{II}}^{(2)}=\frac{H}{2 K} \int_{A} \mathbf{f}^{*(2) \cdot}\left(K_{1}^{(1)} \frac{\partial \mathbf{u}^{(3)}}{\partial a}-K_{\mathrm{I}}^{(3)} \frac{\partial \mathbf{u}^{(1)}}{\partial a}\right) \mathrm{d} A .
\end{aligned}
$$

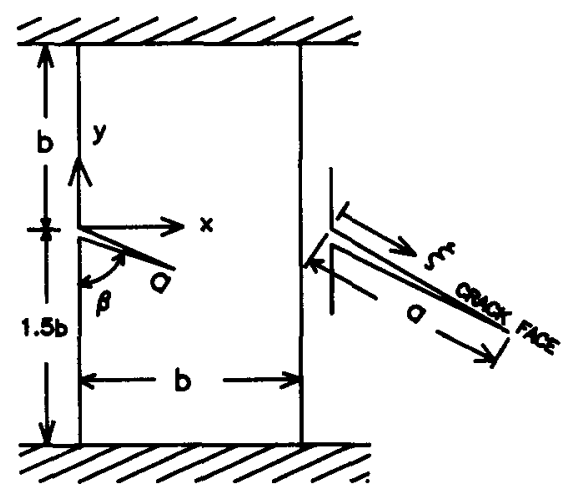

Fig. 13. Geometry and coordinate definitions for the cracked plate with an oblique edge crack. 


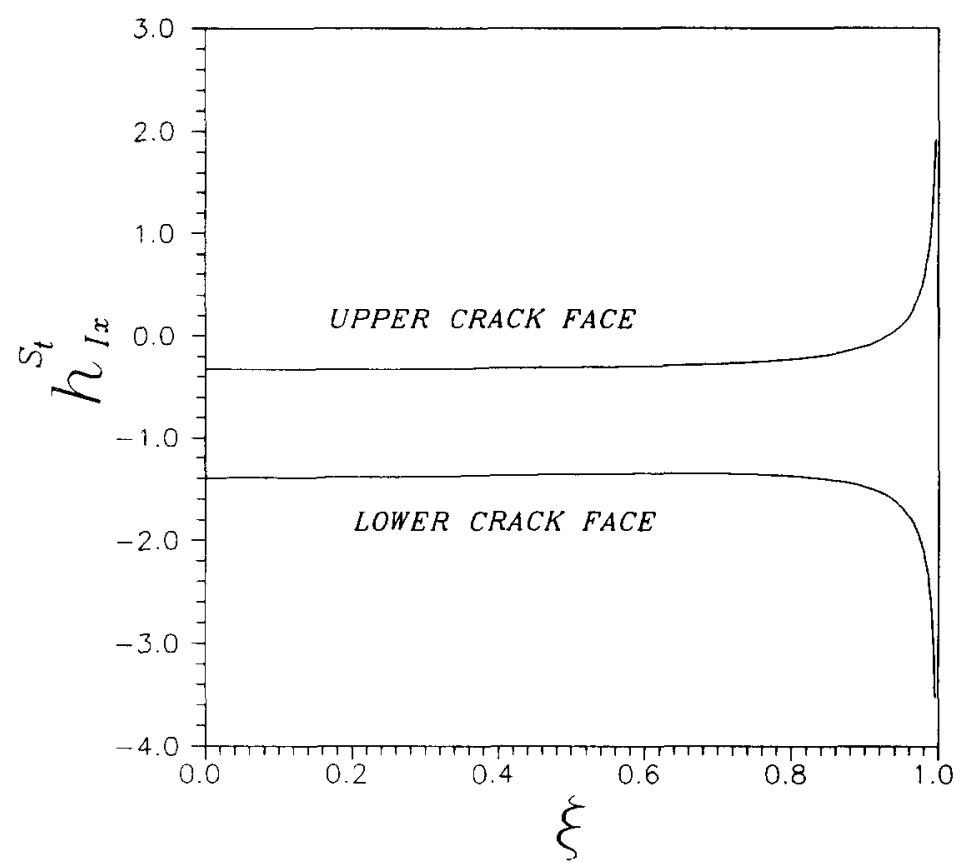

Fig. 14. Crack face weight functions $h_{x}^{S_{t}}$ on upper and lower crack faces.

The body force weight functions for mixed-mode loadings are presented as follows

$$
\begin{aligned}
& \mathbf{h}_{\mathrm{I}}^{f}=\frac{H}{2 K}\left(K_{\mathrm{I}}^{3)} \frac{\partial \mathbf{u}^{(1)}}{\partial a}-K_{\mathrm{I}}^{1)} \frac{\partial \mathbf{u}^{(3)}}{\partial a}\right), \\
& \mathbf{h}_{\mathrm{II}}^{f}=\frac{H}{2 K}\left(K_{\mathrm{I}}^{(1)} \frac{\partial \mathbf{u}^{(3)}}{\partial a}-K_{\mathrm{I}}^{(3)} \frac{\partial \mathbf{u}^{(1)}}{\partial a}\right) .
\end{aligned}
$$

We can see from eqs (10) and (11) that the expressions of the weight functions for the surface traction and body force have the same formulations. The subscript $\mathbf{I}$ in $\mathbf{h} \mathbf{f}$ is used to denote the mode I body force weight function. The mode I weight function vector $h_{f}^{f}$ consists of $h_{1 x}^{f}$ and $h_{1 y}^{f}$ components, which are defined as

$$
\begin{aligned}
& h_{1 . x}^{f}=\frac{H}{2 K}\left(K_{\mathrm{II}}^{(3)} \frac{\partial u_{x}^{(1)}}{\partial a}-K_{\mathrm{II}}^{(1)} \frac{\partial u_{x}^{(3)}}{\partial a}\right), \\
& h_{\mathrm{Iy}}^{f}=\frac{H}{2 K}\left(K_{\mathrm{II}}^{(3)} \frac{\partial u_{y}^{(1)}}{\partial a}-K_{\mathrm{II}}^{(1)} \frac{\partial u_{y}^{(3)}}{\partial a}\right),
\end{aligned}
$$

where $u_{x}^{(1)}, u_{x}^{(3)}$ and $u_{y}^{(1)}, u_{y}^{(3)}$ are the displacement components along the $x$ and $y$ axes, respectively. The corresponding mode II weight functions $h_{\text {IIx }}$ and $h_{1 y}$ have the similar form as eqs (16) and (17), which are

$$
\begin{aligned}
& h_{\mathrm{IIx}}^{f}=\frac{H}{2 K}\left(K_{\mathrm{I}}^{(1)} \frac{\partial u_{x}^{(3)}}{\partial a}-K_{\mathrm{f}}^{3)} \frac{\partial u_{x}^{(1)}}{\partial a}\right), \\
& h_{\mathrm{II} y}^{f}=\frac{H}{2 K}\left(K_{1}^{1)} \frac{\partial u_{y}^{(3)}}{\partial a}-K_{\mathrm{f}}^{(3)} \frac{\partial u_{y}^{(1)}}{\partial a}\right) .
\end{aligned}
$$




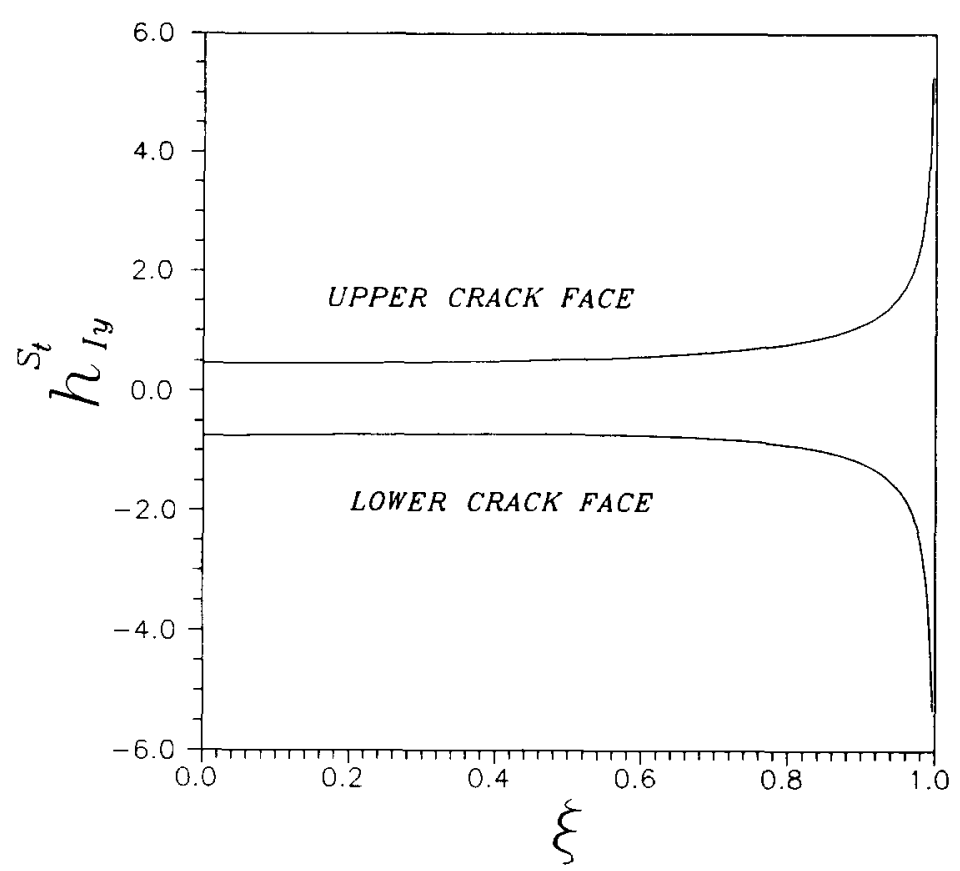

Fig. 15. Crack face weight functions $h_{1 y}^{S t}$ on upper and lower crack faces.

\section{BODY FORCE WEIGHT FUNCTIONS FOR FINITE RECTANGULAR CRACKED} PLATES

In order to obtain the body force weight function, reference stress intensity factors together with the corresponding displacement field must be known. Exact solutions of the displacement field are available for only a very few crack problems. For most cases that are of practical interest, i.e. cracks in finite bodies, exact solutions hardly exist. Hence, the boundary element method by coupling the virtual crack extension technique is used in this study to calculate the body force weight function.

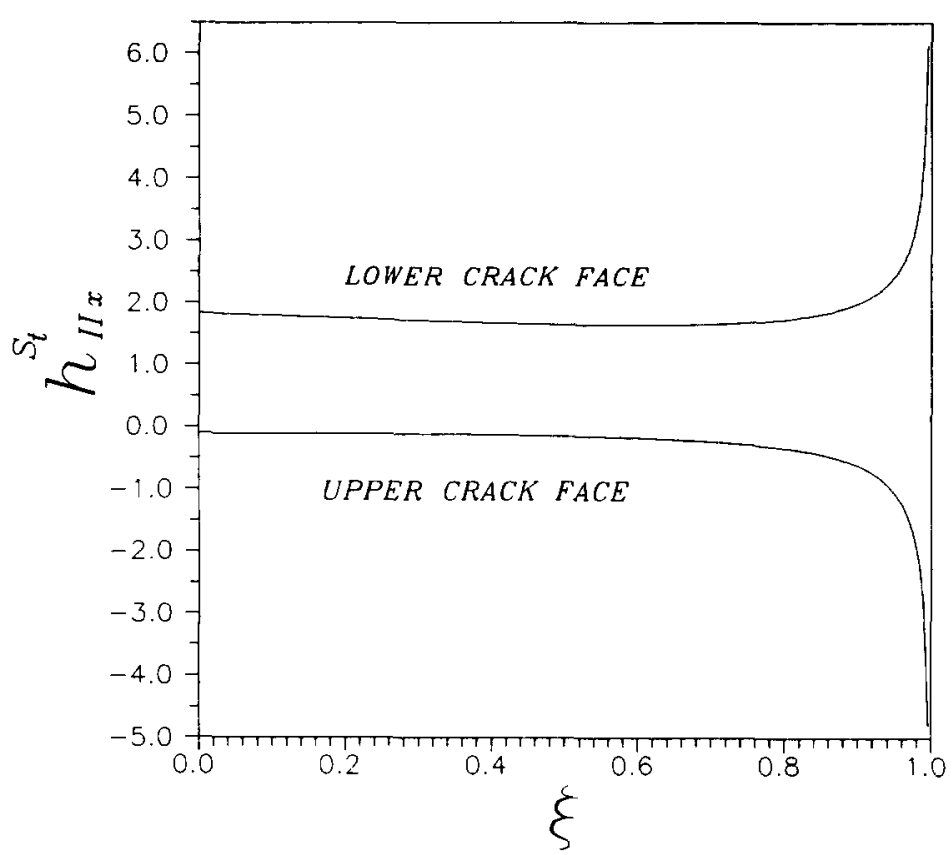

Fig. 16. Crack face weight functions $h_{11 . x}^{S t}$ on upper and lower crack faces. 
function, we obtain the surface traction weight functions along the upper and lower crack faces of the oblique edge crack (Figs 14-17). The square root singular behavior of the crack face weight functions in the crack tip neighborhood is also presented in these figures. These predetermined weight functions along the upper and lower crack faces can be used to evaluate the mixed-mode stress intensity factors of the same crack configuration. A uniformly distributed traction with unit magnitude applied on part of the crack faces for $0.65 \leq \xi \leq 0.8$ in the $y$ direction is analyzed. The mixed-mode stress intensity factors are obtained from the integration of the predetermined weight functions which are plotted in Figs 14-17; the results are $K_{\mathrm{I}}=0.2092, K_{\mathrm{II}}=0.0822$. If the uniformly distributed traction is applied on the oblique crack faces for $0 \leq \xi \leq 0.3$, the induced mixed-mode stress intensity factors are $K_{\mathrm{I}}=0.3217, K_{\mathrm{II}}=-0.00125$.

\section{CONCLUSIONS}

The calculation of stress intensity factors is of great significance to the analysis of structures containing cracks. Stress intensity factors are now available for a wide range of crack configurations and loadings, and have been summarized in well-known handbooks. It is still found inadequate with regard to the needs in practical applications. The weight function serves as a universal function for a given crack geometry and composition; they are independent of the applied loading. The weight function concept is, in fact, Green's function of the stress intensity factors for a cracked body. The weight function for mechanical loading is now a very common method to determine the stress intensity factor. The key advantage of the weight function method is that once the weight function is predetermined for a given crack geometry, accurate stress intensity factors for any other loading conditions for the same crack geometry can be obtained by simple integration. Therefore, the use of the weight function concept can obviate the need for repeated computer computations of stress intensity factors for a given crack geometry under different loading conditions.

In this study, we investigate the body force weight function for a finite rectangular plate with single edge or center crack geometry. The body force weight function is calculated numerically by the boundary element method. Some features of the body force weight function are presented and discussed in this study.

Acknowledgement-The financial support for the authors by the Nation Science Council (Republic of China) through Grant NSC 78-0210-D002-19 is gratefully acknowledged.

\section{REFERENCES}

[1] H. F. Bueckner, A novel principle for the computation of stress intensity factors. ZAMM 50, 529 (1970).

[2] J. R. Rice, Some remarks on elastic crack-tip stress field. Int. J. Solid Structures 8, 751 (1972).

[3] L. B. Freund and J. R. Rice, On the determination of elastodynamic crack tip stress field. Int. J. Solid Structures 10, 411 (1974).

[4] O. L. Bowie and C. E. Freese, Cracked-rectangular sheet with linearly varying end displacements. Engng Fracture Mech. 14, $519(1981)$.

[5] Y. Bortman and L. Banks-Sills, An extended weight function method for mixed-mode elastic crack analysis. J. appl. Mech. 50, 907 (1983).

[6] T. L. Sham and Y. Zhou, Weight functions in two-dimensional bodies with arbitrary anisotropy. Int. J. Fracture 40, 13 (1989).

[7] H. F. Bueckner, Field singularities and related integral representations, in Mechanics of Fracture 1: Methods of Analysis and Solution of Crack Problems (Edited by G. C. Sih), Vol. 239. Noordhoff, Leyden.

[8] J. R. Rice, Three dimensional elastic crack tip interactions with transformation strains and dislocations. Int. J. Solids Structures 21, 781 (1985).

[9] J. R. Rice, Weight function theory for three-dimensional elastic crack analysis, in Fracture Mechanics: Perspectives and Directions (Edited by R. P. Wei and R. P. Gangloff), ASTM STP 1020, 29, Philadelphia.

[10] H. F. Bueckner, Weight functions and fundamental fields for penny-shaped and half-plane crack in three-space. Int. $J$. Solids Structures 23, 57 (1987).

[11] T. L. Sham, A unified finite element method for determining weight functions in two and three dimensions. Int. J. Solids and Structures 23, 1357 (1987).

[12] T. L. Sham and Y. Zhou, Computation of three-dimensional weight functions for circular and elliptical cracks. Int. J. Fracture 40, 51 (1989).

[13] H. Gao, Weight functions for external circular cracks. Int. J. Solids Structures 25, 107 (1989). 


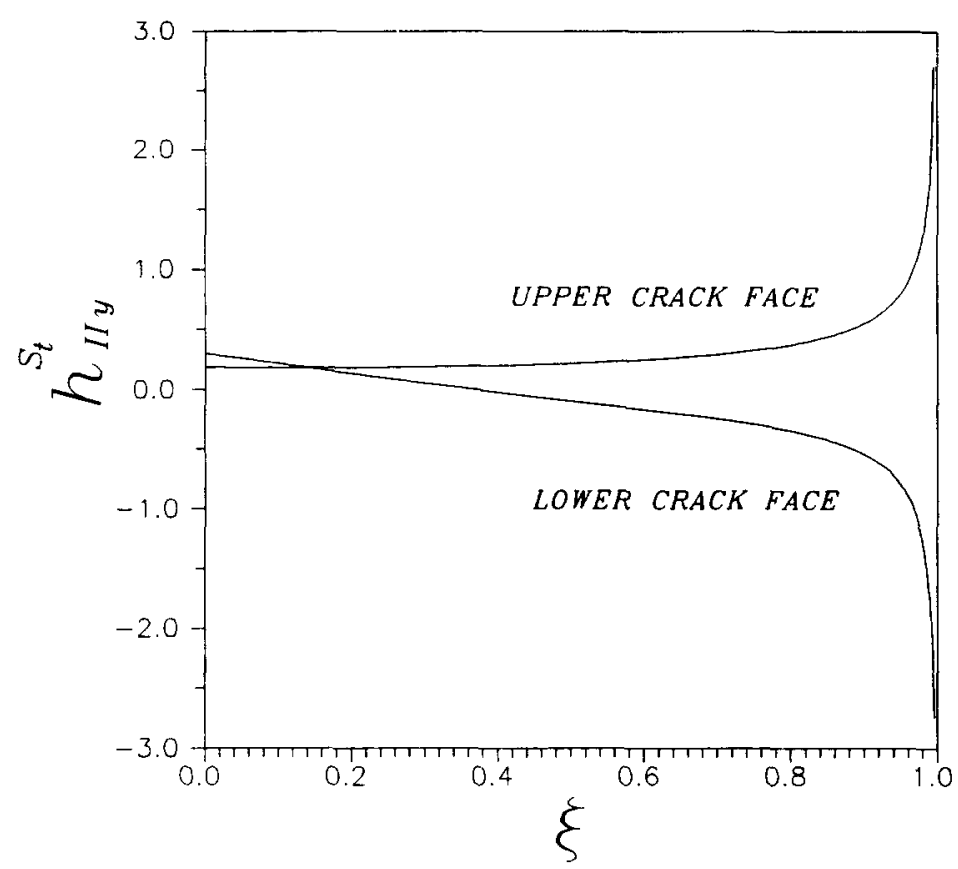

Fig. 17. Crack face weight functions $h_{\mathrm{Ily}}^{\text {st }}$ on upper and lower crack faces.

Before we evaluate the body force weight function, the crack face weight function is investigated in order to make sure that the numerical method used in this study is efficient and accurate. Consider an edge crack in a rectangular plate which is fixed at the top and bottom sides; the configuration is shown in Fig. 2. The crack face weight function is calculated and plotted in Fig. 3 which shows the square root singularity near the crack tip. A symmetric uniformly distributed loading with unit magnitude is applied at the crack faces. The mode I stress intensity factor $K_{1}$ is evaluated by two methods, one is directly calculated and the value is 4.49 , the other one is obtained by using the weight function method and the solution is 4.47 . We can see from this example that very accurate stress intensity factors can be obtained by using the weight function method. We believe that the body force weight function presented in this study can also provide similar accuracy for calculating stress intensity factors as we have for the crack face weight function mentioned above.

We now consider the body force weight function for the cracked body geometry with a fixed boundary (Fig. 2). The body force weight functions of mode I for applying body forces in the $x$-direction are plotted in Fig. 4 . It is shown in this figure that the equal value of the weight function will become straight at the position $y=W$. The variation of the body force weight function as a function of $x$ for different values of $y$ is plotted in Fig. 5. Similar results of body force weight functions for applying body forces in the $y$-direction are plotted in Fig. 6. The corresponding results for different values of $y$ are plotted in Fig. 7. It is shown in Fig. 7 that the weight function behaves as a square root singularity when the applying body force is near the crack tip (i.e. $y$ is small and $x$ approximately zero).

Next we consider a center crack with crack length $2 a(a / W=0.1, W / L=0.1)$ in a finite rectangular plate (Fig. 8). We focus our attention only on the mode I body force weight function for the right crack tip. The body force weight functions for applying body forces in the $x$-direction are shown in Fig. 9. The body force weight functions for different values of $y$ are plotted in Fig. 10. The body force weight functions for applying body forces in the $y$-direction are shown in Fig. 11 . Figure 12 represents the body force weight functions for different values of $y$ and it also shows the singular behavior near the crack tip. Finally, the mixed-mode weight function is evaluated, but we only focus on the crack face weight function. We consider the edge crack geometry with the top and bottom sides of the rectangular plates fixed, the oblique crack angle $\beta=\tan ^{-1} 2$ and $a=1, b=2 \mathrm{a}$ (Fig. 13). Following the same procedure as indicated above for constructing the body force weight 
[14] H. Gao, Application of 3-d weight functions-I. Formulations of crack interactions with transformation strains and dislocations. J. Mech. Phys. Solids 37, 133 (1989).

[15] H. Gao and J. R. Rice, Application of 3-d weight functions-II. The stress field and energy of a shear dislocation loop at a crack tip. J. Mech. Phys. Solids 37, 155 (1989).

[16] C. C. Ma, Z.S. Chang and C. H. Tsai, Weight functions of oblique edge and center cracks in finite bodies. Engng Fracture Mech. 36, 267 (1990).

[17] C. H. Tsai and C. C. Ma, Thermal weight function of cracked bodies subjected to thermal loading. Engng Fracture Mech. 41, 27 (1992).

(Received 14 July 1994) 